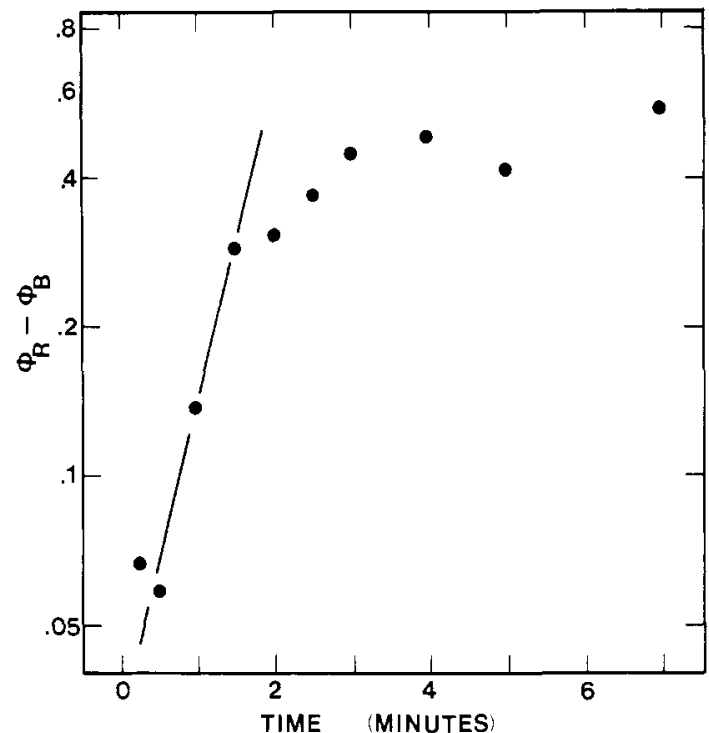

Figure 9. Short-time dependence of the rich-phase polystyrene volume fraction with time of annealing at $423 \mathrm{~K}$.

$1.5 \mathrm{~min}$. Nishi et al. obtained a similar result. ${ }^{2}$

As can be seen in Figure 7, a large change in concentration at very short times does not lead to a large change in the ratio of excimer-to-monomer fluorescence. As a result of this reduced sensitivity, the value of the dominant growth rate $R\left(\beta_{\mathrm{m}}\right)$ may be determined only to within a factor of 2. From the slope of the line drawn through the short-time data in Figure 9, $R\left(\beta_{\mathrm{m}}\right)=0.9 \pm 0.3 \mathrm{~min}^{-1}$. Nishi et al. obtained a growth rate for a 50:50 blend with higher molecular weight components annealed at $403 \mathrm{~K}$ which was an order of magnitude slower.

The diffusion coefficient describing the decomposition process can be calculated provided the wavelength of the dominant concentration fluctuation is known. From optical microscopy, this wavelength has been determined to be of the order of $1 \mu \mathrm{m}^{2}$ From eq 9 , the diffusion coefficient is then found to be of the order of $-10^{-11} \mathrm{~cm}^{2} / \mathrm{s}$.

Finally, the fluorescence results show that phase growth is still taking place at long times where Cahn's model does not hold. From Table I, it appears that the growth rate at long times is several orders of magnitude slower than the rate during the early stages of decomposition.

\section{Conclusions}

The technique of excimer fluorescence can be used to study quantitatively the kinetics of phase separation in polymer blends. For a $10 \%$ PS/PVME blend annealed at $423 \mathrm{~K}$, Cahn's model for spinodal decomposition adequately describes the process at short times, although the fluorescence results indicate that equilibrium is not reached during this period. The growth rate appears to be several orders of magnitude larger at short times than during the later stages of decomposition.

Acknowledgment. This work was supported by the Army Research Office under Contracts DAAG 29-78-C0047 and DAAG 29-82-K-0019.

\section{References and Notes}

(1) McMaster, L. P. Macromolecules 1973, 6, 760.

(2) Nishi, T.; Wang, T. T.; Kwei, T. K. Macromolecules 1975, 8, 227.

(3) McMaster, L. P. Polym. Prepr., Chem. Soc., Div. Polym. Chem. 1974, 15, 254.

(4) Cahn, J. W. J. Chem. Phys. 1965, 42, 93.

(5) Van Aartsen, J. J. Eur. Polym. J. 1970, 6, 919.

(6) Smolders, C. A.; Van Aartsen, J. J.; Steenbergen, A. Kolloid $Z$. Z. Polym. 1971, 243, 14.

(7) Bank, M.; Leffingwell, J.; Thies, C. Macromolecules 1971, 4, 43.

(8) Bank, M.; Leffingwell, J.; Thies, C. J. Polym. Sci., Part A-2 $1972,10,1097$.

(9) Kwei, T. K.; Nishi, T.; Roberts, R. F. Macromolecules 1974 , 7,667 .

(10) Nishi, T.; Kwei, T. K. Polymer 1975, 16, 285

(11) Gelles, R.; Frank, C. W. Macromolecules 1982, 15, 741.

(12) Gelles, R.; Frank, C. W. Macromolecules 1982, 15, 747.

(13) Frank, C. W.; Gashgari, M. A. Macromolecules 1979, 12, 163.

(14) Koningsveld, R.; Staverman, A. J. Kolloid Z. Z. Polym. 1967, $218,114$.

(15) Koningsveld, R.; Staverman, A. J. J. Polym. Sci., Part C 1967, 16,1775 .

(16) Frank, C. W.; Gashgari, M. A. Ann. N.Y. Acad. Ści. 1981, 366, 387.

(17) Semerak, S. N.; Frank, C. W. Macromolecules 1981, 14, 443.

(18) Gashgari, M. A.; Frank, C. W. Macromolecules 1981, 14, 1558.

(19) Semerak, S. N.; Frank, C. W. ACS Adv. Chem., in press.

(20) Fitzgibbon, P. D.; Frank, C. W. Macromolecules 1982, 15, 733.

(21) Birks, J. B., Ed. "Organic Molecular Photophysics"; Wiley: New York, 1975; Vol. 2.

\title{
Calculation of Liquid-Liquid Phase Separation in a Ternary System of a Polymer in a Mixture of a Solvent and a Nonsolvent
}

\author{
Frank W. Altena and C. A. Smolders*
}

Department of Chemical Technology, Twente University of Technology, 7500 AE Enschede, The Netherlands. Received January 4, 1982

\begin{abstract}
A numerical method for the calculation of the binodal of liquid-liquid phase separation in a ternary system is described. The Flory-Huggins theory for three-component systems is used. Binodals are calculated for polymer/solvent/nonsolvent systems which are used in the preparation of asymmetric ultrafiltration or reverse osmosis membranes: cellulose acetate/solvent/water and polysulfone/solvent/water. The values for the binary interaction parameters are taken from literature sources. The effect of a concentration-dependent solvent/nonsolvent interaction parameter is discussed. Although knowledge of the interaction parameters for all compositions in the ternary system is rather poor, fairly good agreement has been found between calculated and experimentally found miscibility gaps when the solvent/nonsolvent parameter is taken to be concentration dependent and the other parameters, the polymer/solvent and the polymer/ nonsolvent interaction parameter, are kept constant.
\end{abstract}

\section{Introduction}

The equilibrium thermodynamic properties of the system polymer/solvent/nonsolvent play an important role in the description of the formation process of an asym- metric reverse osmosis or ultrafiltration membrane. Such membranes are usually prepared by casting a concentrated polymer solution in a thin film on a supporting surface and immersing this film in a nonsolvent bath. Depending on 

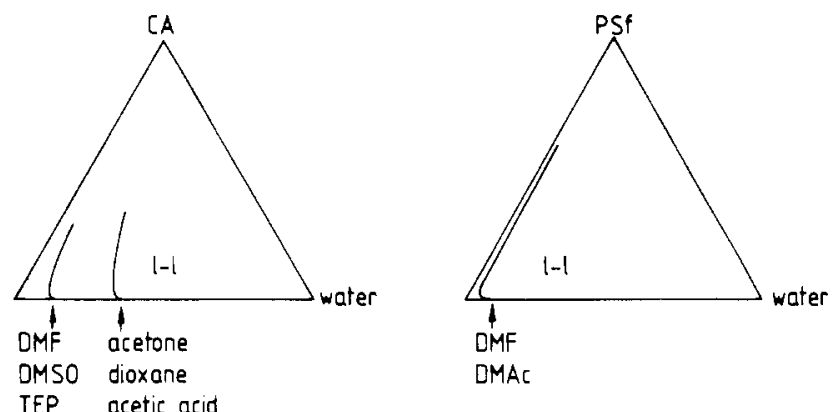

Figure 1. Approximate situation of the liquid-liquid (1-l) phase separation gap in important membrane-forming systems.

the choice of the system, there are three possible results: ${ }^{1,2}$ (i) a uniformly dense film having a porosity comparable to that of the bulk polymer obtained from evaporation procedures, (ii) a porous membrane containing an important void fraction, or (iii) a thin dense top layer on a porous supporting layer. The latter is the desired asymmetric structure. Examples are membranes formed from solutions of cellulose acetate (CA) and polysulfone (PSn) coagulated in water. An example of the first category, a homogeneous, dense CA membrane, is obtained when acetone is used as the solvent. A rather porous membrane is the result if triethyl phosphate (TEP) is used as the solvent. Examples of the third category are membranes from casting solutions of $\mathrm{CA}$ with solvent dioxane, acetic acid, or DMF and solutions of PSn with DMF or DMAc as the solvent. Some of these membranes have reverse osmosis (salt rejection) properties whereas others have typical ultrafiltration properties.

An explanation of these observations should be based on two sets of factors: (I) the equilibrium thermodynamic properties of the three-component system polymer/solvent/nonsolvent, such as liquid-liquid phase separation and gelation, and (II) the exchange of solvent and nonsolvent during membrane formation and the effect on the kinetics of the above-mentioned demixing phenomena. A proper model for the formation process of asymmetric membranes should, of course, include both sets of factors.

An interesting observation concerning the thermody. namic properties of the systems is that systems which require only small amounts of nonsolvent (i.e., $<10 \%$ water) to obtain liquid-liquid phase separation, e.g., CA in TEP or DMF and PSn in DMF or DMAc, give a membrane with ultrafiltration properties. ${ }^{1,3-5}$ If a large amount of water is needed to achieve precipitation ( $\simeq 30 \%$ water), the result is a membrane with reverse osmosis properties, e.g., CA in dioxane, acetic acid, or acetone. These facts are illustrated in Figure 1.

We have suggested before ${ }^{3,6}$ that the porosity of the sublayer can be ascribed to liquid-liquid phase separation, the pores being formed most probably by nucleation and growth of the dilute polymer phase. In this paper we will try to predict binodal liquid-liquid phase separation curves in a few polymer/solvent/nonsolvent systems on the basis of the thermodynamics of polymer solutions.

Recently Cohen et $\mathrm{al}^{2}$ proposed a different model for the formation of porous structures in the coagulation step during membrane formation. Their model is based on diffusion-induced phase separation at the spinodal in the ternary system. In their model, which also includes a solution of the diffusion problem, the location and the form of the spinodal and binodal are of decisive importance. These curves have been calculated on the basis of the Flory-Huggins theory. ${ }^{7}$ The binary interaction parameters that enter the equations have been estimated from liter- ature data. In our opinion, these parameters have not been taken from the best possible sources. As will be shown later the spinodal and binodal depend very strongly on the choice of these parameters. Moreover, the authors do not give details of their calculation procedure. This calculation is not a trivial matter. ${ }^{7,8}$

Tompa ${ }^{8}$ was the first to present calculated phase diagrams for liquid-liquid phase separation in a ternary polymer/solvent/nonsolvent system. Later Koningsveld et al. ${ }^{9}$ extensively studied the influence of the molecular weight distribution on the location of the liquid-liquid phase separation gap and the fractionation of the polymer in this demixing process. In principle, Koningsveld's calculation procedures can be used. The location of the miscibility gap, however, depends primarily on the values of the interaction parameters. For our studies on membrane formation we are at this stage, not interested in the much less pronounced effect of the molecular weight distribution. Knowledge of the values of the binary interaction parameters is still rather poor; at least a strong dependence of the binary parameter on the composition of the system and possibly also ternary interaction parameters must be taken into account. Our aim is to compare calculated miscibility gaps in widely different poly. mer/solvent/nonsolvent systems with experimentally determined phase transition boundaries.

If equations for the chemical potentials of the components are available, the least-squares calculation procedure of Hsu and Prausnitz ${ }^{10}$ seems, in principle, to be appropriate for the calculation of the binodal in a ternary system.

\section{Thermodynamics of Three-Component Systems}

We use the Flory-Huggins theory for polymer solutions ${ }^{7}$ extended to systems with three components by Tompa. ${ }^{8}$ In recent years equation-of-state theories have been formulated as improvements of the Flory-Huggins theory, but application to (polar) ternary systems is scarce and full of limitations; e.g., see Horta. ${ }^{11}$

The Gibbs free energy of mixing is given by the following relation:

$$
\begin{aligned}
\Delta G_{\mathrm{m}} / R T=n_{1} \ln \phi_{1} & +n_{2} \ln \phi_{2}+n_{3} \ln \phi_{3}+ \\
& g_{12}\left(u_{2}\right) n_{1} \phi_{2}+\chi_{13} n_{1} \phi_{3}+\chi_{23} n_{2} \phi_{3}
\end{aligned}
$$

The subscripts refer to nonsolvent (1), solvent (2), and polymer (3). $n_{i}$ and $\phi_{i}$ are the number of moles and the volume fraction of component $i$, respectively. $R$ and $T$ have their usual significance. $\chi_{13}$ is the nonsolventpolymer interaction parameter and $\chi_{23}$ is the solventpolymer interaction parameter. $g_{12}$ is the solvent-nonsolvent parameter and it is assumed to be a function of $u_{2}$, with $u_{2}=\phi_{2} /\left(\phi_{2}+\phi_{1}\right)$ in the notation of Pouchly et al. ${ }^{12}$

The conditions for liquid-liquid equilibrium are

$$
\Delta \mu_{i}^{\prime}=\Delta \mu_{i}^{\prime \prime} \quad i=1,2,3
$$

where $\Delta \mu_{i}$ is the difference between the chemical potential of component $i$ in the mixture and the pure state. The superscript prime and double prime denote the dilute and concentrated phases, respectively.

From eq 1 we derive the following equations for the chemical potentials of the components in the mixture:

$$
\begin{aligned}
& \Delta \mu_{1} / R T=\ln \phi_{1}+1-\phi_{1}-s \phi_{2}-r \phi_{3}+ \\
& \quad\left(g_{12} \phi_{2}+\chi_{13} \phi_{3}\right)\left(\phi_{2}+\phi_{3}\right)-s \chi_{23} \phi_{2} \phi_{3}-\phi_{2} u_{1} u_{2}\left(\mathrm{~d} g_{12} / \mathrm{d} u_{2}\right)
\end{aligned}
$$

$$
\begin{aligned}
& s \Delta \mu_{2} / R T=s \ln \phi_{2}+s-\phi_{1}-s \phi_{2}-r \phi_{3}+ \\
& \quad\left(g_{12} \phi_{2}+\chi_{23} s \phi_{3}\right)\left(\phi_{1}+\phi_{3}\right)-\chi_{13} \phi_{1} \phi_{3}+\phi_{1} u_{1} u_{2}\left(\mathrm{~d} g_{12} / \mathrm{d} u_{2}\right)
\end{aligned}
$$




$$
\begin{aligned}
& r \Delta \mu_{3} / R T=r \ln \phi_{3}+r-\phi_{1}-s \phi_{2}-r \phi_{3}+ \\
& \quad\left(\chi_{13} \phi_{1}+s \chi_{23} \phi_{2}\right)\left(\phi_{2}+\phi_{1}\right)-g_{12} \phi_{1} \phi_{2}
\end{aligned}
$$

The chemical potentials $\Delta \mu_{2}$ and $\Delta \mu_{3}$ are multiplied by, respectively, $s$ and $r ; s$ and $r$ are the ratios of the molar volumes $\nu_{1} / \nu_{2}$ and $\nu_{1} / \nu_{3}$, respectively. The quantities on the left-hand side of eq 3-5 thus defined are of comparable magnitude.

Only binary interaction parameters are considered. Their values either have been taken from the literature $\left(g_{12}=g_{12}\left(u_{2}\right)\right)$ or have been determined from separate experiments $\left(\chi_{13}\right.$ and $\chi_{23}$; at this stage assumed to be constant with change in composition). In the calculations the effect of constant or composition-dependent solvent/nonsolvent $\left(g_{12}\right)$ interaction parameters on liquidliquid phase separation has been investigated.

\section{Method of Computation}

Calculation of the Binodal. Tie lines connect the coexisting phases (points on the binodal curve) that have compositions in the dilute phase $\left(\phi_{1}{ }^{\prime}, \phi_{2}{ }^{\prime}\right.$, and $\left.\phi_{3}{ }^{\prime}\right)$ and in the concentrated phase $\left(\phi_{1}{ }^{\prime \prime}, \phi_{2}{ }^{\prime \prime}\right.$, and $\left.\phi_{3}{ }^{\prime \prime}\right)$. In order to determine the tie line we must determine these six unknowns. Three relations between the variables are given by eq $3-5$. The material balance equations $\left(\sum \phi_{i}=1\right.$ for the dilute and the concentrated phase) give another two equations. By choosing one variable (in our case $\phi_{3}{ }^{\prime}$ ) and substituting the material balance equations into (3)-(5), we reduce the problem to computation of three unknowns from three nonlinear transcedental equations. As independent variables we have chosen $\phi_{2}{ }^{\prime \prime}, \phi_{3}{ }^{\prime \prime}$, and $\phi_{2}{ }^{\prime}$.

The numerical procedure is essentially the same as that of Hsu and Prausnitz. ${ }^{10}$ They used a least-squares procedure, which appears to be effective. The objective function that has to be minimized is

$$
F=\sum f_{i}^{2}
$$

with

$$
\begin{gathered}
f_{1}=\Delta \mu_{1}{ }^{\prime}-\Delta \mu_{1}{ }^{\prime \prime} \\
f_{2}=s\left(\Delta \mu_{2}{ }^{\prime}-\Delta \mu_{2}{ }^{\prime \prime}\right) \\
f_{3}=r\left(\Delta \mu_{3}{ }^{\prime}-\Delta \mu_{3}{ }^{\prime \prime}\right)
\end{gathered}
$$

and the chemical potentials are given by eq 3-5. Expressed in this way, the residuals $f_{i}$ are of comparable size.

The coexisting compositions are found by an iterative process. We have used the numerical procedure given by Gill and Murray. ${ }^{13}$ This procedure uses first (and second) derivatives of the expressions for the chemical potentials. In this way, considerable improvements were obtained as compared with the calculation procedure used by Hsu: no penalty functions are needed to avoid a trivial solution; scaling of the volume fractions is not necessary; the starting point of the iteration process is less sensitive than in the method used by Hsu.

Convergence was considered to be attained when $F<$ $10^{-10}$. The CPU time (DEC 10) required for the calculation of 20-50 tie lines in the ternary diagram was about 1-3 s, depending on whether concentration-dependent $g_{12}$ parameters were used or not. The complete miscibility gap is obtained by varying the chosen variable, the polymer concentration in the dilute phase $\phi_{3}$, by a factor 10 .

Calculation of the Spinodal. The equation for the spinodal is 8

$$
G_{22} G_{33}=\left(G_{23}\right)^{2}
$$

where

$$
G_{i j}=\partial^{2} \Delta G_{\mathrm{m}} / \partial \phi_{i} \partial \phi_{j}
$$

It follows, using eq 1

$$
\begin{aligned}
& G_{22}=\frac{1}{\phi_{1}}+\frac{1}{\nu_{2} \phi_{2}}-2 g_{12}+2 g_{12}{ }^{\prime}\left(u_{1}-u_{2}\right)+g_{12}{ }^{\prime \prime} u_{1} u_{2} \\
& G_{33}=\frac{1}{\phi_{1}}+\frac{1}{\nu_{3} \phi_{3}}-2 \chi_{13}+2 u_{2}{ }^{2}\left(1+u_{1}\right) g_{12}{ }^{\prime}+u_{2}{ }^{3} u_{1} g_{12}{ }^{\prime \prime}
\end{aligned}
$$

$G_{23}=\frac{1}{\phi_{1}}-\chi_{13}+\frac{\chi_{23}}{\nu_{2}}-g_{12}-u_{1} u_{2} g_{12}^{\prime}-u_{1} u_{2}^{2} g_{12}^{\prime \prime}$

where $g_{12}{ }^{\prime}=\partial g_{12} / \partial u_{2}$ and $g_{12}{ }^{\prime \prime}=\partial^{2} g_{12} / \partial u_{2}^{2}$. We assumed $\nu_{1}=1$. For the case of constant interaction parameters, the equations reduce to the equation given by Tompa: ${ }^{8}$

$$
\begin{aligned}
& \sum \nu_{i} \phi_{i}-2 \sum \nu_{i} \nu_{j}\left(\chi_{i}+\chi_{j}\right) \phi_{i} \phi_{j}+ \\
& 4 \nu_{1} \nu_{2} \nu_{3}\left(\chi_{1} \chi_{2}+\chi_{1} \chi_{3}+\chi_{2} \chi_{3}\right) \phi_{1} \phi_{2} \phi_{3}=0
\end{aligned}
$$

where

$$
\begin{aligned}
& 2 \chi_{1}=\chi_{12}+\chi_{13}-\chi_{23} / \nu_{2} \\
& 2 \chi_{2}=\chi_{12}+\chi_{23} / \nu_{2}-\chi_{13} \\
& 2 \chi_{3}=\chi_{13}+\chi_{23} / \nu_{2}-\chi_{12}
\end{aligned}
$$

When the interaction parameters are known and one variable is chosen (e.g., $\phi_{3}$ ), substitution of the material balance equation $\sum \phi_{i}=1$ in eq 8 will give one equation with one variable to be solved. This is done by using the same numerical procedure as has been used for the calculation of the binodal.

\section{Evaluation of the Binary Function $\boldsymbol{g}_{12}$}

The function $g_{12}$ is needed at the temperature at which cloud point curves have been obtained. The parameters are calculated from literature data on $G^{\mathrm{E}}$, generally obtained from vapor pressure experiments. For a two-component system $G^{\mathrm{E}}$ is related to the Gibbs free energy of mixing by the equation

$$
G^{\mathrm{E}}=\Delta G_{\mathrm{m}}-R T\left(x_{1} \ln x_{1}+x_{2} \ln x_{2}\right)
$$

where $x_{1}$ and $x_{2}$ are the mole fractions of the nonsolvent (1) and the solvent (2). For $1 \mathrm{~mol}$ of mixture

$$
\Delta G_{\mathrm{m}} / R T=x_{1} \ln \phi_{1}+x_{2} \ln \phi_{2}+g_{12} x_{1} \phi_{2}
$$

From eq 14, $g_{12}$ is calculated as a function of $\phi_{2}$.

Only for a few solvent/water systems are experimental data on $G^{\mathrm{E}}$ available (see compilation of literature references by Wisniak and Tamir ${ }^{14}$ ). In Table I the sources are listed from which data on $G^{\mathrm{E}}$ have been taken.

The binary parameter $g_{12}$ calculated from these data is given for the different solvents in Figure 2. For acetic acid we have used data of Haase. ${ }^{24}$

The magnitude of $g_{12}$ and its variation with concentration influence the thermodynamic properties of a ternary system to a great extent; see, e.g., Aminabhavi. ${ }^{25}$

\section{Evaluation of Binary Parameters $\chi_{13}$ and $\chi_{23}$}

The interaction parameter $\chi_{13}$ is determined from equilibrium swelling measurements carried out by Mul$\mathrm{der},{ }^{26}$ for instance, at different temperatures. We have used the value of 1.4 for the interaction parameter for $\mathrm{CA} /$ water and the value of 3.7 for $\mathrm{PSn} /$ water $\left(25^{\circ} \mathrm{C}\right)$.

The solvent/polymer parameter $\chi_{23}$ is taken from work done in our laboratory. ${ }^{27}$ We determined this parameter using osmometry. For $\mathrm{CA} /$ acetone and $\mathrm{CA} /$ dioxane we have found the values 0.45 and 0.40 , respectively, at $10 \mathrm{wt}$ $\%$ polymer and $25^{\circ} \mathrm{C}$. In this paper we do not consider the concentration dependence of this parameter. For other 


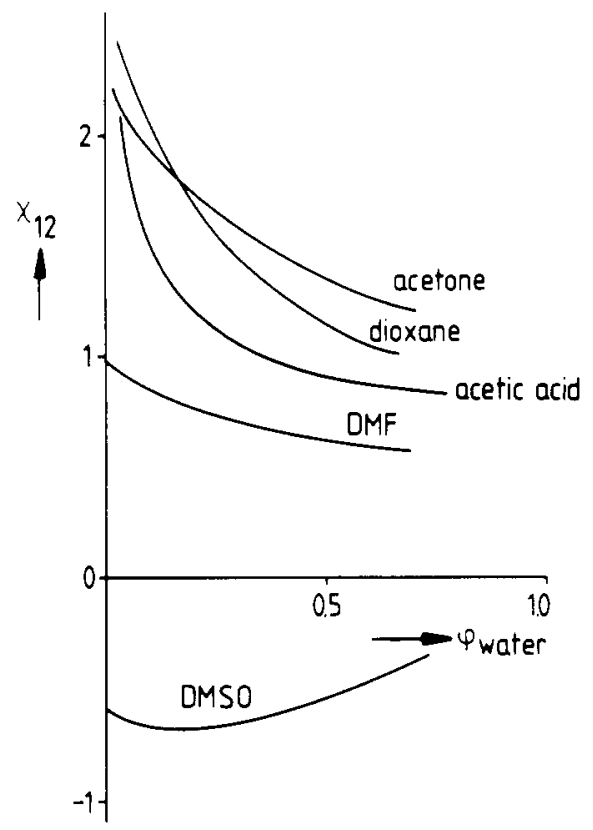

Figure 2. Concentration-dependent interaction parameters in binary solvent/water systems.

polymer/solvent combinations there are no data available.

\section{Results and Discussion}

General. The predicted polymer concentration in the dilute phase rapidly approaches a very small value. For each successive tie line given in the figures, the polymer concentration in the dilute phase is a factor 10 lower if one moves to the right. In some figures tie lines have been

(a)

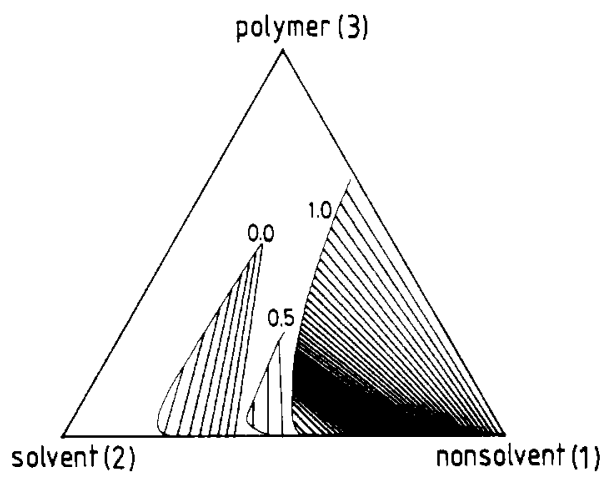

(c)

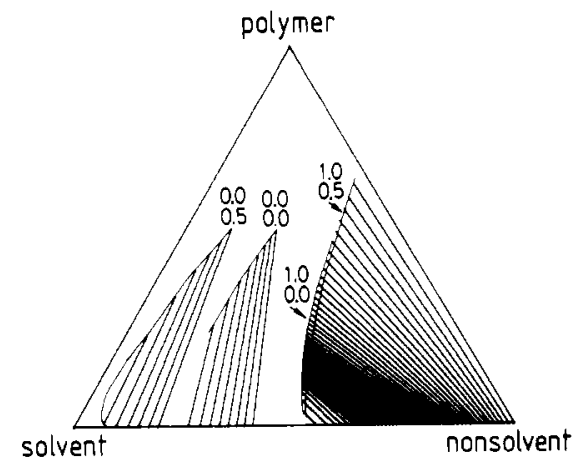

Table I

Literature Sources on $G^{\mathrm{E}}$ of Solvent/Water Mixtures

\begin{tabular}{ll}
\hline \multicolumn{1}{c}{ solvent } & \multicolumn{1}{c}{ ref } \\
\hline dioxane & Kortüm, ${ }^{15}$ Goates, ${ }^{16}$ Malcolm $^{17}$ \\
acetone & Orye, ${ }^{18}$ Othmer $^{19}$ \\
DMF & Saphon ${ }^{20}$ \\
$\mathrm{Me}_{2}$ SO & Lam, $^{21}$ Chan $^{22}$ \\
actic acid & Gieskes, ${ }^{23} \mathrm{Haase}^{24}$ \\
TEP & no data available \\
DMAc & no data available \\
${ }^{a}$ Conflicting data.
\end{tabular}

calculated for a polymer concentration $\phi_{3}{ }^{\prime}$ of $10^{-30}$. Of course, this value can have no physical meaning.

Tompa ${ }^{8}$ calculated tie lines for a simple case: $\nu_{1}=\nu_{2}$; $\chi_{12}=\chi_{13}=1.5$ and $\chi_{23}=0$. The results of our calculations with these values for the parameters agree completely with his phase diagram.

Constant Interaction Parameters. Our procedure allows for arbitrarily chosen values of the interaction parameters $\chi_{i j}$ and molar volume ratios $\nu_{i} / \nu_{j}$. All practical systems that we consider have a large ratio of solvent and nonsolvent molar volumes; i.e., $\nu_{1} / \nu_{2} \simeq 0.2$. For the polymer volume ratio, we have chosen $\nu_{1} / \nu_{3}=0.002$.

First, we will consider the case of changing the solvent in the system (thus changing $\chi_{12}$ ) while keeping the polymer-solvent and polymer-nonsolvent parameters constant. At two fixed values of $\chi_{13}, 1$ and 1.5, the effect of changing the solvent-nonsolvent parameter can be seen from Figure 3, parts $a$ and $b$. Increasing the $\chi_{12}$ parameter results in a quite drastic change in the location of the miscibility gap. It appears that a low tendency of mixing of solvent and nonsolvent, as expressed by a large value of $\chi_{12}$, favors the mixing of the ternary system: more

(b)

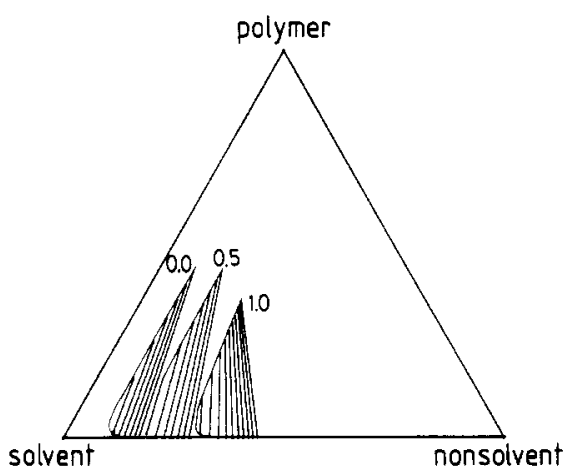

(d)

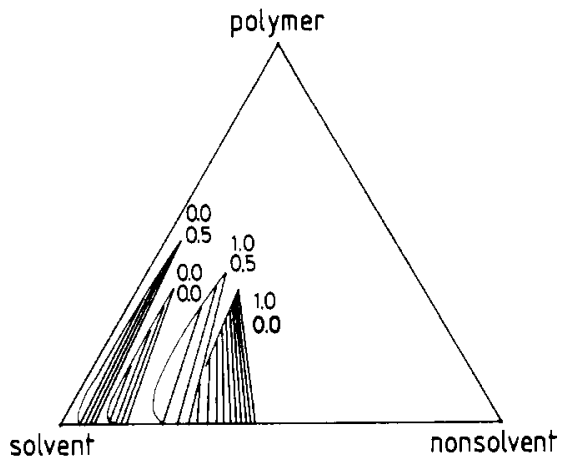

Figure 3. Tie lines and binodals in polymer/solvent/nonsolvent systems for constant interaction parameters. Effect of varying $\chi_{12}$ (values given in the figure) at (a) $\chi_{13}=1.0$ and (b) $\chi_{13}=1.5$. Effect of varying $\chi_{23}$ (lower number at each curve) at certain values of $\chi_{12}$ (upper number) for (c) $\chi_{13}=1.0$ and (d) $\chi_{13}=1.5$. 
Table II

Concentration-Dependent Solvent/Nonsolvent Interaction Parameters

\begin{tabular}{|c|c|c|c|c|c|c|}
\hline \multirow[b]{2}{*}{ solvent } & \multirow[b]{2}{*}{$\nu_{1} / \nu_{2}\left(25^{\circ} \mathrm{C}\right)$} & \multicolumn{5}{|c|}{$g_{12}=a+b \phi_{2}+c \phi_{2}{ }^{2}+d \phi_{2}{ }^{3}+e \phi_{2}{ }^{4}$} \\
\hline & & $a$ & $b$ & $c$ & $d$ & $e$ \\
\hline $\begin{array}{l}\text { dioxane } \\
\text { acetone } \\
\mathrm{DMF} \\
\mathrm{Me}_{2} \mathrm{SO} \\
\text { acetic acid }\end{array}$ & $\begin{array}{l}0.2092 \\
0.2442 \\
0.2336 \\
0.2540 \\
0.3151\end{array}$ & $\begin{array}{r}0.92 \\
1.10 \\
0.50 \\
-0.25 \\
\text { not co }\end{array}$ & $\begin{array}{c}-0.69 \\
-0.42 \\
0.04 \\
-0.69 \\
\text { further }\end{array}$ & $\begin{array}{l}7.15 \\
4.09 \\
0.80 \\
1.15\end{array}$ & $\begin{array}{c}-12.91 \\
-6.70 \\
-1.20 \\
-2.9\end{array}$ & $\begin{array}{l}8.17 \\
4.28 \\
0.82 \\
2.09\end{array}$ \\
\hline
\end{tabular}

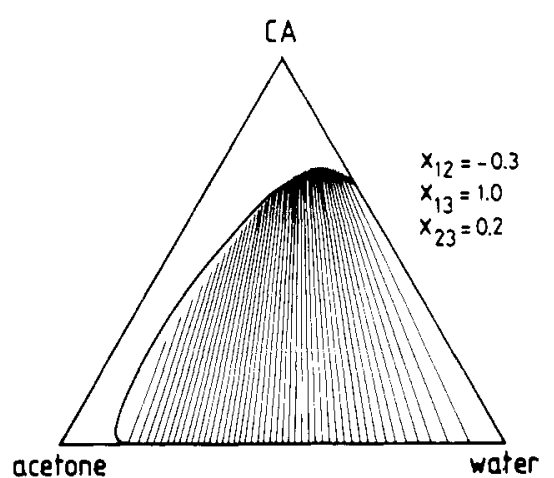

Figure 4. Comparison of calculated binodal with literature (Cohen et al., ${ }^{2}$ solid curve) for the system CA/acetone/water. Values of interaction parameters are given.

nonsolvent is needed for liquid-liquid phase separation. Another interesting point to observe is the polymer concentration in the concentrated phase, which is much higher at low $\chi_{12}$ than at high $\chi_{12}$ for the same degree of penetration of the liquid-liquid phase separation region (here measured as $\phi_{3}{ }^{\prime \prime}$ at the same $\phi_{3}{ }^{\prime}$ at curves for different $\chi_{12}$ ). The tendency of the location of the miscibility gap to move to the right in the ternary diagrams is not yet understood on physical grounds.

Secondly, we consider the influence of a changing solvent quality for the polymer $\left(\chi_{23}\right)$ on the location of the tie lines. Results are presented in parts $c$ and d of Figure 3. At low values of $\chi_{12}$ the effect of increasing $\chi_{23}$ is considerable, but in opposite direction of increasing $\chi_{12}$, whereas at high values of $\chi_{12}$, especially at $\chi_{12}=1$, there is only a small effect on the location of the liquid-liquid phase separation region. A higher value of $\chi_{23}$ decreases the amount of nonsolvent necessary for phase separation. This is also the result of choosing a higher $\chi_{13}$ parameter. Qualitatively, these effects are not difficult to understand. The effect of changing $\chi_{13}$ can be seen more clearly by comparing parts $a$ and $b$ of Figure 3.

Comparison with Phase Diagram for Cellulose Acetate/Acetone/Water from Cohen et al. Cohen et $\mathrm{al}^{2}$ have calculated the binodal for the system CA/ acetone/water and CA/acetic acid/water. For the acetone system they used the following interaction parameters: $\chi_{12}$ $=-0.30, \chi_{13}=1$, and $\chi_{23}=0.2$ with $\nu_{1}: \nu_{2}: \nu_{3}=1: 4: 500$. We calculated tie lines of the liquid-liquid phase separation with the same set of parameters. Our results and the diagram of Cohen are compared in Figure 4. There are small differences in the location of the binodals. Cohen gives no details of his calculation procedure, so that the exact origin of these differences cannot be traced.

In our opinion, a much better choice of the interaction parameters is possible from literature data. Swelling measurements indicate a CA/water parameter of about 1.4. The CA/acetone parameter takes the value of about 0.45 . More important, however, is the influence of the solvent/nonsolvent parameter. From Figure 2 we conclude that $\chi_{12}$ has at least a large positive value, and not a value of -0.3 as assumed by Cohen. Cohen et al. based their

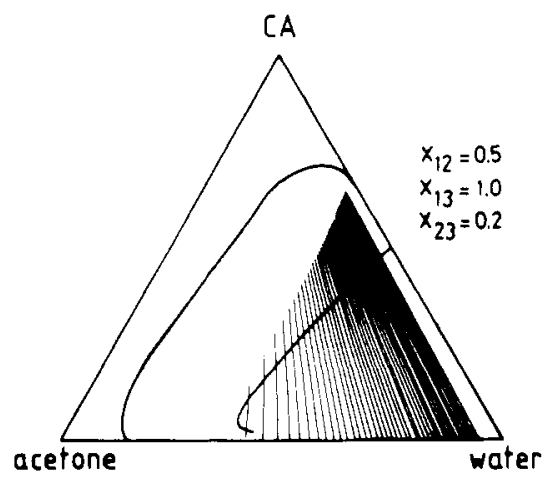

Figure 5. Effect of change in acetone/water interaction parameter from -0.3 to +0.5 . The left full curve has been taken from Cohen et al. The right full curve is the spinodal.

estimate of the $\chi_{12}$ parameter only on the enthalpy of mixing and not on the free energy of mixing as they should have done (see, e.g., Aminabhavi et al. ${ }^{25}$ ).

In Figure 5 the phase diagram is given for a positive value of $\chi_{12}$, i.e., 0.5 . The other parameters are kept at the values that Cohen used. The spinodal has also been calculated for this case. The result could have a serious effect on the conclusion of the study of Cohen et al. that phase separation at the spinodal produces the porous substructure of CA membranes cast from acetone solutions. However, in order to ascertain any effect, the above calculations of the phase diagram should be supplemented with a calculation of the diffusion path followed by the system during coagulation, as has been done by Cohen et al. ${ }^{2}$

Concentration-Dependent $\boldsymbol{g}_{12}$ Parameters. Keeping the other interaction parameters constant, we now consider a composition-dependent $g_{12}$ parameter (cf. Figure 2); $g_{12}$ is calculated from literature data on $G^{\mathrm{E}}$. In our calculations we use the data for each solvent from the first mentioned author in Table I. There can be considerable differences between data of different authors, e.g., in $G^{\mathbf{E}}$ for acetic acid/water. ${ }^{23,24}$ These differences are reflected in the results of the calculation of the binodals.

For the $g_{12}\left(\phi_{2}\right)$ function a fourth-order polynomial relation has been chosen; the coefficients were found by using a least-squares method. The function fits the $g_{12}$ values calculated from the experimental data within $3 \%$. Using this $g_{12}$ function, we calculated $\Delta G_{\mathrm{m}}$ (eq 14) over the full composition range to check if demixing in some range of the solvent/nonsolvent mixture without polymer is predicted. This would, of course, have a serious effect on the subsequent calculations. For instance, the $g_{12}$ function calculated from the data of Goates ${ }^{16}$ on dioxane/water predicts liquid-liquid phase separation at about a 50/50 mixture of dioxane/water. The coefficients of the interaction parameter function $g_{12}$ are given in Table II.

Comparison with Experimental Data. Cellulose Acetate (CA). There is not much information on the location of the liquid-liquid phase separation gap in CA systems. Frommer ${ }^{1}$ and Guillotin ${ }^{4}$ give values for the precipitation concentration for water at about $20 \%$ poly- 


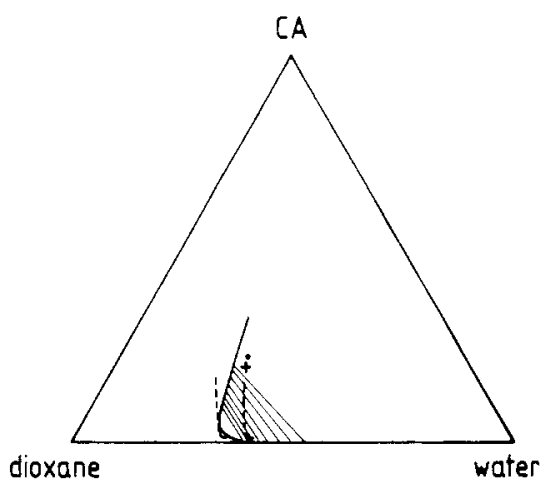

Figure 6. Calculated binodal for CA/dioxane/water (full curve). Dashed curves from cloud point measurements at 20 (left) and $60^{\circ} \mathrm{C}$ (right). ${ }^{31}$ Single-point data from Frommer ${ }^{1}(+)$ and Guillotin ${ }^{4}(\cdot)$ at $20^{\circ} \mathrm{C}$.

mer. We have determined cloud points for $\mathrm{CA}$ /dioxane/ water $^{3}$ and also for CA/DMF/water (Figure 8). Strathmann $^{28}$ and Lemoyne ${ }^{5}$ give data on CA/acetone/water that deviate from those of the above-mentioned sources.

CA/Dioxane/Water. Experimental points and curves are given in Figure 6 . The full curve is calculated with the $g_{12}$ function from Table II and with $\chi_{13}=1.4$ and $\chi_{23}$ $=0.4$.

The experimental data from Frommer and Guillotin do not agree with our measurements. The reason for this deviation is not clear.

We note a fairly good agreement in the position of experimental and calculated curves. The set of interaction parameters used appears to describe the demixing of the system reasonably well.

The only computed effect of introducing a smaller $\chi_{23}$ value is that the polymer concentration in the concentrated phase is higher at about the same solvent/nonsolvent ratio as compared to the situation drawn in the diagram.

When a smaller value of $\nu_{1} / \nu_{2}$ is used (a value of 0.002 means a molecular weight of 9000 , whereas the $C A$ often used has a molecular weight of about 20000-30000), no effect on the location of the miscibility gap is found. The distance between the tie lines, differing a factor 10 in the polymer concentration in the dilute phase, then becomes much smaller.

A similar agreement in location between experimental cloud point curves and theoretical binodals can be obtained by taking an effective, constant, $\chi_{12}$ value of 1.1 instead of $g_{12}\left(\phi_{2}\right)$. In view of the expected strong concentration dependence of $\chi_{12}$ (Table II), a constant value of $\chi_{12}$, however, is not acceptable.

CA/Acetone/Water. The experimental information on liquid-liquid phase separation in $\mathrm{CA}$ /acetone/water is not very distinct. Strathmann ${ }^{28}$ has given so-called tie lines. These lines cannot be real tie lines because he ignores the obscuring effect of gelation of the concentrated phase at some stage of the separation into two liquid phases in equilibrium. Lemoyne ${ }^{5}$ gives a gelation line in the ternary diagram, but he also gives tie lines that connect a dilute phase with a concentrated phase that lie within the gelation region of the system. Our calculated diagram ( $g_{12}$ from Table II, $\chi_{13}=1.4$ and $\chi_{23}=0.45$ ) agrees quite well with the data points from Frommer and Guillotin; see Figure 7 .

CA/DMF/Water. Results are presented in Figure 8. Our own experimental data confirm the results of Frommer and Guillotin. The calculated curves for polymer/solvent interaction parameters of 0.0 and 0.4 (the precise values are not known) do not agree well with the experimental data.

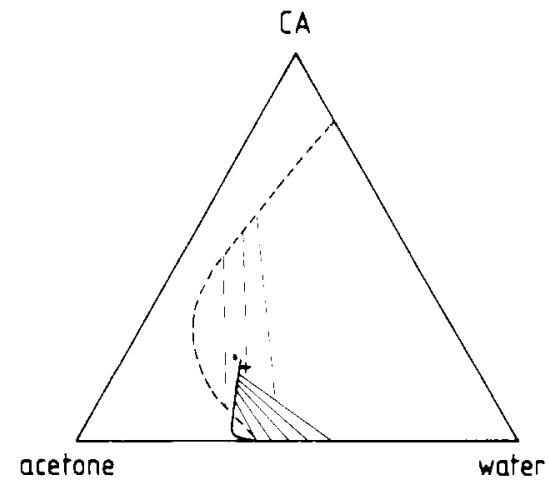

Figure 7. Calculated binodal and tie lines for $\mathrm{CA}$ /acetone/water. Literature data from Frommer ( + ) and Guillotin (.); Strathmann ${ }^{28}$ and Lemoyne ${ }^{5}$ : binodal $(---)$ and tie lines $(-\cdot-)$.

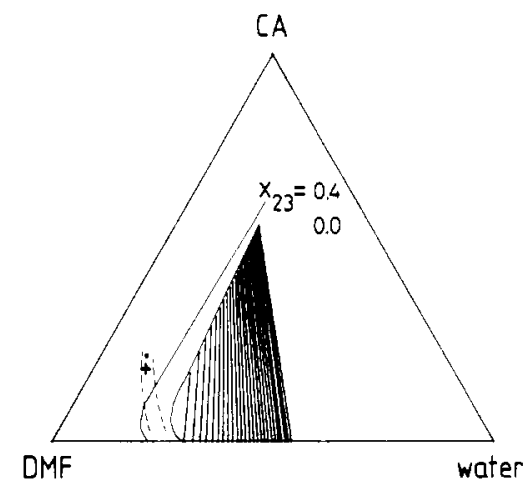

Figure 8. Calculated binodals for CA/DMF/water. Dashed lines from our cloud point experiments at 20 (left) and $40^{\circ} \mathrm{C}$ (right). Literature data from Frommer $(+)$ and Guillotin $(\cdot)\left(\right.$ at $\left.20^{\circ} \mathrm{C}\right)$.

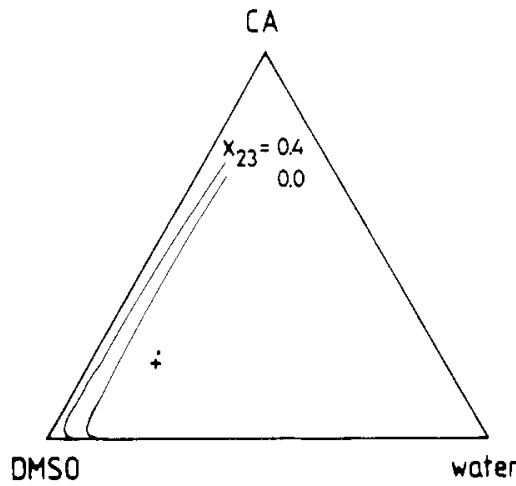

Figure 9. Calculated binodals for $\mathrm{CA} / \mathrm{Me}_{2} \mathrm{SO} /$ water for two values of $\chi_{23}$. Literature data from Frommer $(+)$ and Guillotin $(\cdot)$.

CA $/ \mathbf{M e}_{2} \mathrm{SO} /$ Water. Again two calculated binodals are given, $\chi_{23}=0.0$ and 0.4 ; see Figure 9 . The agreement in position between calculated curves and experimental points is much worse than with the other solvents considered. One reason might be the peculiar behavior of the binary solvent/nonsolvent system itself. This is the only system with a strong negative value for $\chi_{12}$.

Polysulfone (PSn). Demixing data for PSn/DMF/ water have been given by Broens, ${ }^{3}$ Wijmans, ${ }^{29}$ and Coplan. ${ }^{30}$ Only a small amount of water, usually a few percent, is needed to achieve precipitation.

From Figure 10 it is clear that the demixing behavior is mainly determined by $\chi_{13}$ (we used a value of 3.7). Large variations in $\chi_{12}$ and $\chi_{23}$ only have a small absolute effect on the precipitation concentration. Changing the solvent, for instance, taking DMAc instead of DMF, would have this small effect. A more precise comparison with experimental data can be carried out if $\chi_{23}$ and $\chi_{12}$ are known 


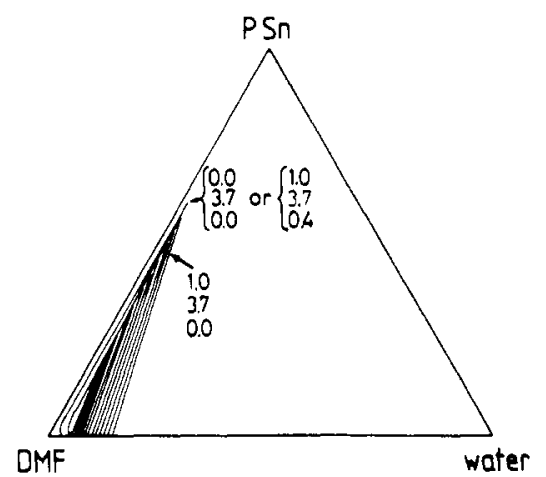

Figure 10. Binodals in PSn/DMF/water at different values of interaction parameters $\chi_{12} / \chi_{13} / \chi_{23}$ (given in figure).

for both solvents. The relative influence of a change of the solvent on the membrane-forming properties is known to be large. ${ }^{29}$

Summarizing the results we can make the following remarks:

A method of computation of phase diagrams has been developed that works quite well.

On the basis of literature information the solvent/nonsolvent interaction parameter $g_{12}$ is assumed to be composition dependent. From our study we conclude that $G^{\mathrm{E}}$, the excess free energy of mixing, has to be considered and not only the enthalpy of mixing. ${ }^{1}$ Other binary parameters have been kept constant. At this stage a more precise study does not seem relevant without detailed information about the ternary interaction parameters in the system and the molecular weight distribution.

A change in the solvent of the membrane-forming system is reflected in a change in $g_{12}$. The qualitative and quantitative effects on the position of the liquid-liquid phase separation gap can be predicted reasonably well. This is shown for CA systems for which binary parameters are known.

For PSn a large polymer/nonsolvent interaction parameter determines the demixing behavior to a great extent. Changes in other binary parameters have only a small effect on the precipitation concentration.

\section{Conclusions}

For the calculation of the binodal in a system of polymer/solvent/nonsolvent we have developed a method of computation that works well.
Introduction of a concentration-dependent solvent/ nonsolvent interaction parameter gives a reasonable agreement between the experimentally found miscibility gap and the calculated binodal of the liquid-liquid phase separation.

\section{References and Notes}

(1) Frommer, M. A.; Lancet, D. In "Reverse Osmosis Membrane Research"; Lonsdale, H. K.; Podall, H. E., Eds.; Plenum Press: New York, 1972; p 85.

(2) Cohen, C.; Tanny, G. B.; Prager, S. J. Polym. Sci., Polym. Phys. Ed. 1979, 17, 477 .

(3) Broens, L.; Altena, F. W.; Smolders, C. A.; Koenhen, D. M. Desalination 1980, 32, 33

(4) Guillotin, M.; Lemoyne, C.; Noël, C.; Monnerie, L. Desalination $1977,21,165$.

(5) Lemoyne, C.; Halary, J. L.; Friedrich, C.; Noël, C.; Monnerie, L. J. Appl. Polym. Sci. 1980, 25, 1883.

(6) Koenhen, D. M.; Mulder, M. H. V.; Smolders, C. A. J. Appl. Polym. Sci. 1977, 21, 199.

(7) Flory, P. J. "Principles of Polymer Chemistry"; Cornell University Press: Ithaca, NY, 1953; p 541.

(8) Tompa, H. "Polymer Solutions"; Butterworths: London, 1956; $\mathrm{p} 182$.

(9) Koningsveld, R. Chem. Zvesti 1972, 26, 263. Br. Polym. J. $1975,7,435$.

(10) Hsu, C. C.; Prausnitz, J. M. Macromolecules 1974, 7, 320.

(11) Horta, A. Macromolecules 1979, 12, 785.

(12) Zivný, A.; Pouchlý, J. J. Polym Sci., Part A-2 1972, 10, 1467.

(13) Gill, P. E.; Murray, W. SIAM J. Numer. Anal. 1978, 15, 977 (available in the NAG library).

(14) Wisniak, J.; Tamir, A. "Mixing and Excess Thermodynamic Properties"; Elsevier: Amsterdam, 1978.

(15) Kortüm, G.; Valent, V. Ber. Bunsenges. Phys. Chem. 1977, 81, 752.

(16) Goates, J. R.; Sullivan, J. R. J. Phys. Chem. 1958, 62, 188.

(17) Malcolm, G. N.; Rowlinson, J. S. Trans. Faraday Soc. 1957, 53, 921.

(18) Orye, R. V.; Prausnitz, J. M. Ind. Eng. Chem. 1965, 57, 18

(19) Othmer, D. F. Ind. Eng. Chem. 1952, 44, 1872

(20) Saphon, S.; Bittrich, H. J. Z. Phys. Chem. (Leipzig) 1973, 252, 113.

(21) Lam, S. Y.; Benoit, R. L. Can. J. Chem. 1974, 52, 718.

(22) Chan, T. C.; Van Hook, W. A. J. Solution Chem. 1976, 5, 107.

(23) Gieskes, J. T. Th. Can. J. Chem. 1965, 43, 2448.

(24) Haase, R.; Pehlke, M.; Dücker, K. H. Z. Naturforsch., A 1973, 28,1740 .

(25) Aminabhavi, T. M.; Munk, P. Macromolecules 1979, 12, 607

(26) Mulder, M. H. V., to be published.

(27) Altena, F. W. Eur. Polym. J., submitted for publication.

(28) Strathmann, H.; Scheible, P.; Baker, R. W. J. Appl. Polym. Sci. $1971,15,811$.

(29) Wijmans, J. G., private communication.

(30) Coplan, M. J.; Davis, R. B.; Beale, J. K. NTIS PB 287990 , Springfield, VA, 1978.

(31) Altena, F. W.; Smolders, C. A. J. Polym. Sci., Polym. Symp. 1981, No. 69, 1. 